\title{
RNA interference-mediated knockdown of Aurora-B alters the metastatic behavior of A549 cells via modulation of the phosphoinositide 3-kinase/Akt signaling pathway
}

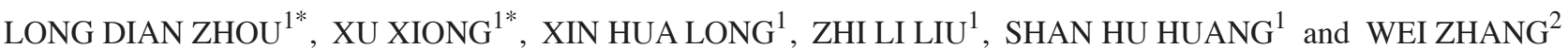 \\ Departments of ${ }^{1}$ Orthopedics and ${ }^{2}$ Respiratory Medicine, The First Affiliated Hospital of Nanchang University, \\ Nanchang, Jiangxi 330006, P.R. China
}

Received January 29, 2014; Accepted July 15, 2014

DOI: $10.3892 / 01.2014 .2464$

\begin{abstract}
Accumulating evidence has revealed that an elevated expression level of Aurora-B is associated with metastasis in various types of malignant tumor. However, it is currently unclear whether this molecule is involved in non-small lung cancer (NSCLC) metastasis, and the molecular mechanisms associated with Aurora-B and metastasis remain unknown. In the present study, in order to investigate whether Aurora-B is involved in the development and metastasis of NSCLC, the Aurora-B protein expression in NSCLC tissues was detected by immunohistochemistry and its association with metastasis was analyzed. The results revealed that the expression levels of the Aurora-B protein in tissues obtained from NSCLC patients with lymph node metastasis were significantly higher than those without metastatic disease. Furthermore, the effect of Aurora-B inhibition on A549 cell migration and invasion, as well as the activity of the phosphoinositide 3-kinase (PI3K)/Akt signaling pathway was evaluated. Aurora-B was inhibited in the A549 cells using short hairpin RNA, and the cell migration and invasion rates were investigated using wound healing and Transwell invasion assays. In addition, the expression of the main proteins in the PI3K/Akt/nuclear factor $-\kappa \mathrm{B}(\mathrm{NF}-\kappa \mathrm{B})$ signaling pathway, and matrix metalloproteinase (MMP)-2 and -9 were measured by western blot analysis. The results demonstrated that cell migration and invasion were decreased
\end{abstract}

Correspondence to: Dr Wei Zhang, Department of Respiratory Medicine, The First Affiliated Hospital of Nanchang University, 17 Yong Wai Zheng Street, Nanchang, Jiangxi 330006, P.R. China

E-mail: zhangwei8869@126.com

Dr Shan Hu Huang, Department of Orthopedics, The First Affiliated Hospital of Nanchang University, 17 Yong Wai Zheng Street, Nanchang, Jiangxi 330006, P.R. China

E-mail: hsh869@126.com

${ }^{*}$ Contributed equally

Key words: non-small cell lung cancer, Aurora B, metastasis, phosphoinositide 3-kinase/Akt signaling pathway as a result of silencing Aurora-B. Furthermore, the activity of the PI3K/Akt/NF- $\mathrm{B}$ signaling pathway and the expression of MMP-2 and -9 protein were suppressed by silencing Aurora-B. The results of the present study indicate that the knockdown of Aurora-B suppresses A549 cell invasion and migration via the inhibition of the PI3K/Akt signaling pathway in vitro and thus, targeting Aurora-B may present a potential treatment strategy for NSCLC.

\section{Introduction}

Lung cancer is the leading cause of cancer-associated mortality worldwide (1). Approximately $80 \%$ of lung cancers are non-small cell lung carcinomas (NSCLC), for which surgery represents the major curative treatment. However, only $30 \%$ of NSCLC patients are able to receive surgery. Furthermore, in the majority of cases, the disease is overtly or covertly metastatic on presentation and consequently is currently incurable. Although chemotherapy has been increasingly adopted for advanced NSCLC treatment, the five-year survival rate of NSCLC remains $<15 \%$ and has not improved in recent years. Therefore, the development of more efficacious targeted therapeutic agents for NSCLC is required.

Aurora B is one of the major protein kinases involved in the execution and fidelity of mitosis. Aurora-B is a member of the chromosomal passenger complex and is involved in numerous mitotic functions, including chromosome-microtubule interactions, sister chromatid cohesion, the spindle-assembly checkpoint mechanism and cytokinesis. Previous studies have shown that Aurora-B is considered to be an important anti-tumor target (2-6). Li et al (7) revealed that the downregulation of Aurora-B inhibited proliferation and metastasis, induced $\mathrm{G}_{2} / \mathrm{M}$ phase arrest in clear cell renal cell carcinoma cells and exerted antitumor activity in an SN12C xenograft model. Furthermore, previous studies have shown that the nuclear Aurora-B expression level is significantly associated with metastasis in tumors (8-12). However, whether Aurora-B is involved in NSCLC metastasis remains unclear.

In the present study, the effect of Aurora-B inhibition on A549 cell invasion and migration was investigated in vitro. In addition, the effect of silencing Aurora-B on the phosphoinositide 3-kinase (PI3K)/Akt/nuclear factor- $\kappa \mathrm{B}(\mathrm{NF}-\kappa \mathrm{B})$ 
signaling pathway was investigated. The aim of the present study was to investigate whether knockdown of Aurora B inhibits A549 cell invasion and migration via downregulation of the PI3K/Akt/NFKB signaling pathway in vitro.

\section{Materials and methods}

Cell cultures. The human NSCLC A549 cell lines (Shanghai Cell Bank, Chinese Academy of Sciences, Shanghai, China) were cultured in Dulbecco's modified Eagle's medium (HyClone, Thermo Fisher Scientific, Waltham, MA, USA) with $10 \%$ fetal bovine serum (FBS; Sigma-Aldrich, St. Louis, $\mathrm{MO}, \mathrm{USA}$ ) and incubated at $37^{\circ} \mathrm{C}$ in an atmosphere of $5 \% \mathrm{CO}_{2}$.

Patients and specimens. A total of 67 NSCLC tissue samples were obtained from patients who underwent surgery at The First Affiliated Hospital of Nanchang University (Nanchang, China). A total of 28 cases exhibited lymph node metastasis, and 39 cases were identified without lymph node metastasis. The lymph node metastasis survey was performed via histopathological detection of the lymph node. No patient had a history of receiving any prior therapeutic treatment with anti-tumor agents or via radiotherapy. Informed consent was obtained from all patients and the study was approved by the ethics committee of Nanchang University (Nanchang, China).

Immunohistochemical analysis. Histological sections $(4 \mu \mathrm{m})$ were stained with hematoxylin and eosin and detected by immunohistochemical analysis, which was performed using the streptavidin-peroxidase procedure. Briefly, antigen retrieval was performed by heating the deparaffinized rehydrated sections in $10 \mathrm{~mm}$ citrate buffer ( $\mathrm{pH}$ 6.0; Abcam, Cambridge, UK) for $20 \mathrm{~min}$, followed by blocking with $10 \%$ goat serum (Sigma-Aldrich). Next, sections were incubated overnight at $4^{\circ} \mathrm{C}$ with the primary rabbit anti-Aurora-B monoclonal antibody (Abcam) at a final dilution of 1:500. For negative controls, sections were incubated with phosphate-buffered saline (PBS; Beijing Solarbio Science \& Technology Co., Ltd., Beijing, China) rather than with antibodies. Following three washes with PBS, the sections were incubated with biotinylated secondary monoclonal mouse anti-rabbit antibody $(1: 1,000$; Santa Cruz Biotechnology, Inc., Santa Cruz, CA, USA) for $40 \mathrm{~min}$, followed by incubation with horseradish peroxidase (HRP)-conjugated streptavidin (Beijing Solarbio Science \& Technology Co., Ltd.) for $30 \mathrm{~min}$. The sections were then chemiluminescence-stained and counterstained using hematoxylin. The stained sections were subsequently scored by two pathologists that were blinded to the clinicopathological features of the patients. The staining intensity was analyzed (by examining $\geq 500$ cells from five representative areas), the expression level of Aurora-B was evaluated and the intensity scores were recorded as follows: No staining, 0; weak staining, 1; moderate staining, 2; and intense staining, 3. According to the percentage of tumor cells exhibiting a positive expression of Aurora-B, the following percentage scores were used: $0 \%$, score $0 ;<10 \%$, score $1 ; 47-50 \%$, score 2 ; $51-80 \%$, score 3 ; and $81-100 \%$, score 4 . The final score was averaged according to the scores that were determined by the two pathologists according to the percentage scores. The intensity score was then added to the percentage score; a final score of $<4$ was defined as (-), 4 as (+), 5 as (++) and $\geq 6$ as (+++).
Recombinant lentivirus-vector (LV) construction and cell transfection. The human mRNA sequence (NM_004217) encoding the Aurora-B protein was obtained from GenBank (http://www.ncbi.nlm.nih.gov/pubmed/). An interfering short hairpin RNA (shRNA) targeting Aurora-B was designed and synthesized, as well as a negative shRNA, which served as a negative control. shRNA sequences were inserted into the LV, GV115 (Aurora-B/LV and negative $[\mathrm{Neg}] / \mathrm{LV}$ ) and transfected into the A549 cells (multiplicity of infection =20). The transfection efficiency was evaluated using a fluorescence microscope (BX51M, Olympus Corporation, Tokoyo, Japan).

Quantitative polymerase chain reaction ( $q P C R)$ assays. Total RNA from the A549 cells was extracted using TRIzol reagent (Invitrogen Life Technologies, Carlsbad, CA, USA). qPCR was used to detect Aurora-B mRNA expression, with $\beta$-actin serving as the endogenous reference gene. All procedures were performed according to the manufacturer's instructions and the following primers were used: Forward, 5'-AGAAGGAGAACTCCTACCCCT-3' and reverse, 5'-CGCGTTAAGATGTCGGGTG-3' for Aurora-B (product length, 202 bp). Six independent experiments were performed over numerous days.

Western blot analysis. Total protein from the cells was extracted using radio-immunoprecipitation lysis buffer containing $60 \mathrm{lg} / \mathrm{ml}$ phenylmethylsulfonyl fluoride [Tiangen Biotech (Beijing) Co., Ltd., Beijing, China]. The protein concentration was determined by Bradford assay (Sigma-Aldrich). Western blot analysis was conducted using the following primary monoclonal anti-human antibodies: Rabbit anti-Aurora-B IgG, (1:200), rabbit anti-PI3K IgG (1:1,000), rabbit anti-phosphorylated (p)Akt IgG (1:800) goat anti-Akt IgG (1:1,000), rabbit anti-NF- $\mathrm{B}(\mathrm{p} 65) \mathrm{IgG}$ (1:400), rabbit anti-matrix metalloproteinase (MMP)-2 (1:1,000) and rabbit anti-MMP-9 IgG (1:1,000), which were all purchased from Abcam and mouse anti-glyceraldehyde 3-phosphate dehydrogenase (1:5,000; Santa Cruz Biotechnology, Inc., Santa Cruz, CA, USA) and the corresponding mouse anti-rabbit, mouse anti-goat and goat anti-mouse monoclonal secondary antibodies (ZSGB-BIO, Beijing, China). The immune complexes were detected using the pro-light Streptavidin-HRP kit (Pierce Biotechnology, Inc., Rockford, IL, USA). Six independent experiments were performed over numerous days.

Transwell assays. The invasion of A549 cells was measured using the BD BioCoat ${ }^{\mathrm{TM}}$ BD Matrigel ${ }^{\mathrm{TM}}$ Invasion Chamber (BD Biosciences, Franklin Lakes, NJ, USA) according to the manufacturer's instructions. The medium in the lower chamber contained $5 \%$ fetal calf serum as a source of chemoattractants. The cultures were rinsed with PBS and replaced with fresh quiescent medium alone or containing $10 \% \mathrm{FBS}$, following which the cells were incubated at $37^{\circ} \mathrm{C}$ for $24 \mathrm{~h}$. Cells that had passed through the matrigel-coated membrane were stained using Diff-Quik (Sysmex, Kobe, Japan) and images were captured using a camera (S200, Canon Inc., Tokoyo, Japan) and an inverted microscope (Olympus Corporation). Cell migration was quantified by direct microscopic visualization and counting. The rate of invasion was calculated by counting three fields per membrane and presented as the mean of six independent experiments performed over various days. 

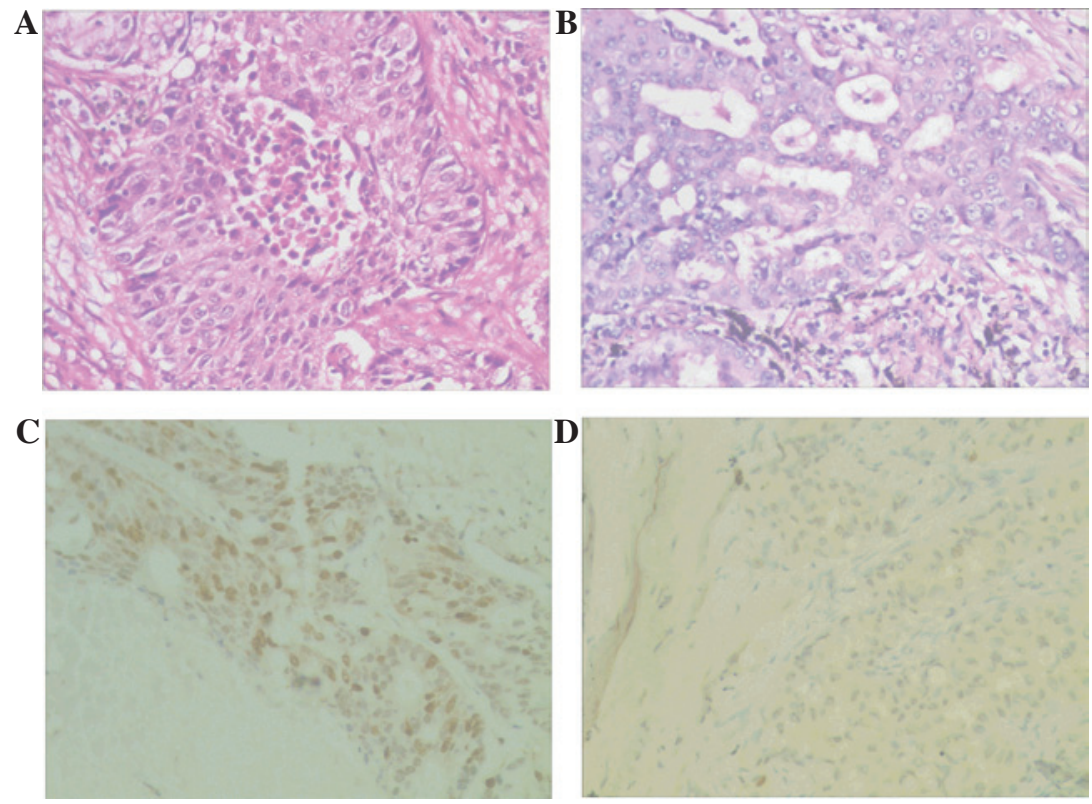

Figure. 1. Aurora-B expression in NSCLC patient samples with and without lymph node metastasis (magnification, $\mathrm{x} 400$ ). Representative images of hematoxylin and eosin-stained NSCLC tissues (A) with and (B) without lymph node metastasis and immunohistochemical staining for Aurora-B in NSCLC tissues (C) with and (D) without lymph node metastasis. NSCLC, non-small cell lung carcinoma.

Wound healing assay. Cell migration was analyzed by determining the ability of the cells to move into a cellular space in a two-dimensional in vitro wound healing assay. Briefly, cells were grown to confluence in six-well tissue culture plates at a density of $\sim 5 \times 10^{6}$ cells/well. The cells were then denuded by dragging a rubber policeman (Thermo Fisher Scientific Inc., Rockford, IL, USA) through the center of the plate. Cultures were then rinsed with PBS and replaced with fresh quiescent medium alone or containing 10\% FBS, following which the cells were incubated at $37^{\circ} \mathrm{C}$ for $24 \mathrm{~h}$. Images were captured at 0 and $24 \mathrm{~h}$, and the migrated distance was measured in five independent wound sites per group. Six independent experiments were performed over numerous days.

Statistical analysis. All data are presented as the mean \pm standard deviation. The two independent-samples t-test was used to analyze the difference between Aurora-B protein expression levels in NSCLC patients with and without lymph node metastasis. $\mathrm{P}<0.05$ was considered to indicate a statistically significant difference. All analyses were performed using SPSS version 13.0 (SPSS, Inc., Chicago, IL, USA).

\section{Results}

Aurora-B protein may be involved in lymph node metastasis in NSCLC. Aurora-B was expressed in the nucleus (Fig. 1) and the positive expression rate in the samples with metastatic disease was $82.1 \%$ (23/28), however, in those without lymph node metastasis the rate was only $43.6 \%$ (17/39); the difference was identified to be significant $(\mathrm{P}<0.05)$ (data not shown). These results indicated that Aurora-B may be involved in lymph node metastasis in NSCLC.

Recombinant LV inhibits Aurora-B expression in A549 cells. A549 cells were transfected with the recombinant LV targeting
A

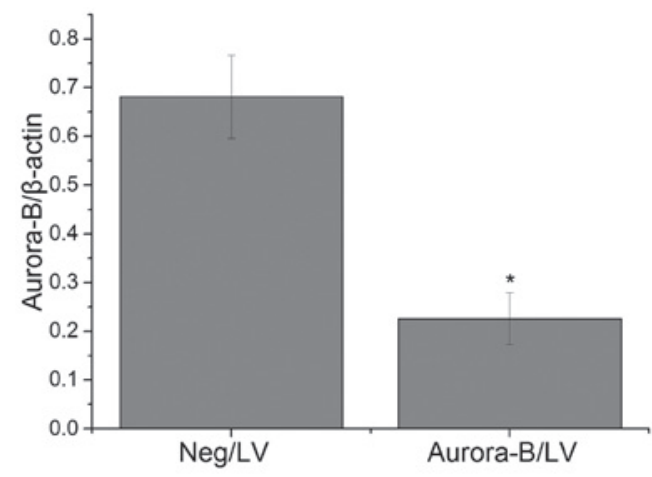

B

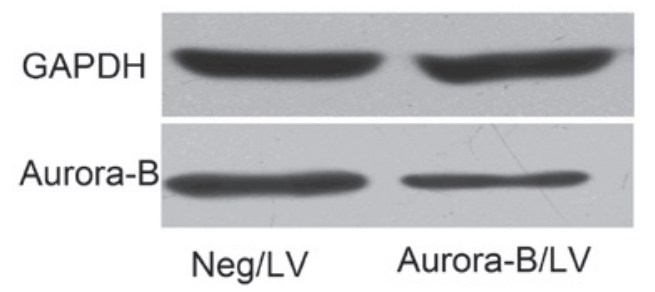

Figure 2. The inhibitory effect of the recombinant LV on the expression level of Aurora-B (A) mRNA (the columns represent the mean of six experiments and the line represents the standard deviation) and (B) protein in A549 cells. ${ }^{*} \mathrm{P}<0.05$ vs. the $\mathrm{Neg} / \mathrm{LV}$ group. Aurora-B/LV, recombinant lentivirus-vector targeting Aurora-B; Neg/LV, negative lentivirus-vector; GAPDH, glyceraldehyde 3-phosphate dehydrogenase.

Aurora-B. qPCR and western blot analysis revealed that the level of Aurora-B protein expression was significantly lower in cells transfected with Aurora-B/LV compared with in those transfected with Neg/LV (Fig. 2).

Inhibition of Aurora-B suppresses A549 cell migration. To investigate whether Aurora-B affects cellular migration, an in vitro wound healing assay was performed. The results showed that the migration rates of cells transfected with 


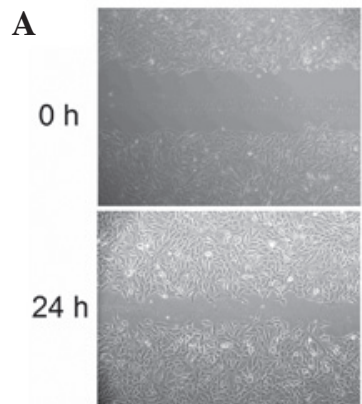

$\mathrm{Neg} / \mathrm{LV}$

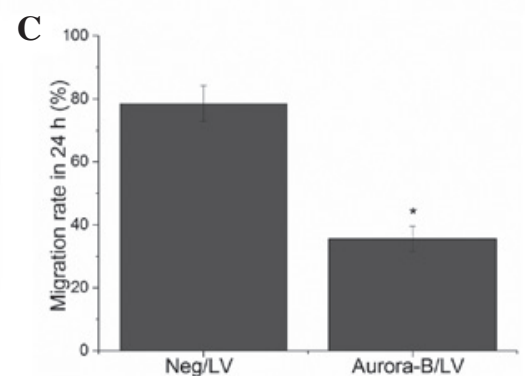

B

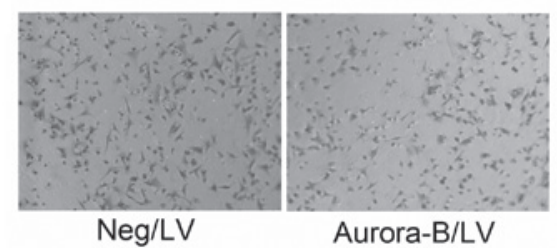

D

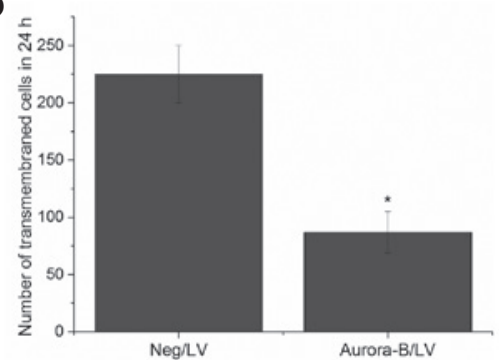

Figure 3. The effect Aurora-B inhibition on A549 cell migration and invasion in vitro. Representative images of six experiments of (A) wound healing and (B) Transwell invasion assays are demonstrated for each group. (C) The migration rate of cells was $35.6 \pm 3.98 \%$ in the Aurora-B/LV transfected group and $78.5 \pm 5.66 \%$ in the Neg/LV transfected group. (D) The number of transmembrane cells was $87 \pm 18$ cells per high power field in the Aurora-B/LV transfected group and $225 \pm 25$ cells per high power field in the $\mathrm{Neg} / \mathrm{LV}$ transfected group. The columns represent the mean of six experiments and the line demonstrates the standard deviation; " $\mathrm{P}<0.05$ vs. the Neg/LV group. Aurora-B/LV, recombinant lentivirus-vector targeting Aurora-B; Neg/LV, negative lentivirus-vector.

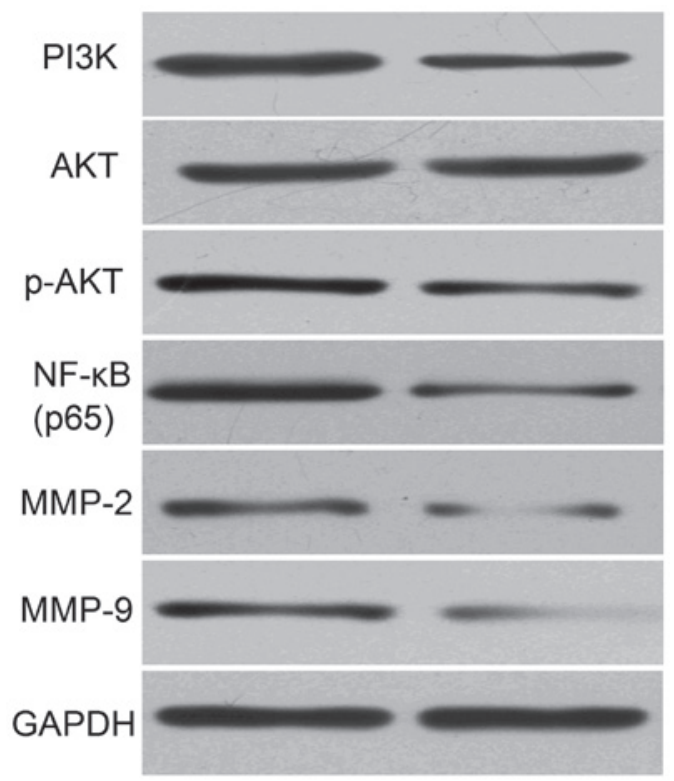

Figure 4. Aurora-B inhibition decreased PI3K/Akt/NF- $\mathrm{KB}$ signaling in A549 cells. Right column, cells transfected with Aurora-B/LV; left column, cells transfected with Neg/LV PI3K, phosphoinositide 3-kinase; NF-kB, nuclear factor- $\mathrm{\kappa}$; p, phosphorylated; MMP, matrix metalloproteinase; GAPDH, glyceraldehyde 3-phosphate dehydrogenase; Neg/LV, negative lentivirus-vector.

Aurora-B/LV and Neg/LV were $35.6 \pm 3.98 \%$ and $78.5 \pm 5.66 \%$, respectively. The difference was identified to be significant (Fig. 3A and B). These results indicated that Aurora-B inhibition may suppress A549 cell migration in vitro.
Inhibition of Aurora-B suppresses A549 cell invasion. A Transwell assay was performed to evaluate the effect of Aurora-B inhibition on the invasion of A549 cells. The results revealed that the number of transmembrane cells was lower in the cells that had been transfected with Aurora-B/LV (87 \pm 18 cells per high power field) when compared with that of the cells transfected with $\mathrm{Neg} / \mathrm{LV}(225 \pm 25$ cells per high power field $[\mathrm{P}<0.05]$; Fig. $3 \mathrm{C}$ and $\mathrm{D}$ ). These results indicated that the inhibition of Aurora-B may suppress the invasion of A549 cells in vitro.

Silencing Aurora-B inhibits PI3K/Akt/NF- $\mathrm{B}$ B signaling in A549 cells. In order to investigate the effect of Aurora-B inhibition on the activity of the PI3K/Akt signaling pathway in A549 cells, the expression levels of PI3K, Akt, p-Akt, NF- $\mathrm{KB}$ (p65) as well as the MMP-2 and -9 proteins was measured using western blot analysis. The results revealed that the level of PI3K, p-Akt, NF- $\mathrm{B}$ (p65), MMP-2 and -9 protein expression in cells transfected with Aurora-B/LV was significantly lower than that of cells transfected with Neg/LV (Fig. 4). This indicated that the inhibition of Aurora-B may inhibit the activity of the PI3K/Akt/ $\mathrm{NF}-\mathrm{kB}$ signaling pathway in A549 cells.

\section{Discussion}

Aurora kinases are serine/threonine kinases, which are crucial for cell cycle control and mitosis. Three Aurora kinase family members (A, B and C) have been identified in mammals and are expressed at maximal levels during mitosis. Aurora-B, a component of the chromosome passenger complex, is located on 
the chromosome arms during prophase, and at the centromeres during the prometaphase and metaphase. Aurora-B subsequently localizes to the midbody during cytokinesis. Previous studies have shown Aurora-B to be overexpressed in numerous types of cancer $(9,10,13)$. In the present study, the expression levels of Aurora-B protein in NSCLC tissues were detected by immunohistochemistry, which revealed that the Aurora-B protein was expressed in the nucleus. Furthermore, the positive expression rate of Aurora-B protein in the NSCLC tissues with lymph node metastasis was significantly higher when compared with the tissue samples without lymph node metastasis. These results are consistent with those reported by Takeshita et al (9) and Wang et al (14). The results of the present study indicated that Aurora-B may be involved in the development and progression of lymph node metastasis, and may present a novel diagnostic and therapeutic target for NSCLC.

Various studies have revealed that the inhibition of Aurora-B blocked cell proliferation and induced cell apoptosis in a variety of tumors (15-17). These findings highlighted Aurora-B as a potential molecular target for cancer treatment. Notably, recent studies have shown that the upregulation of Aurora-B expression was associated with tumor cell metastasis, and the downregulation of Aurora-B inhibited cell invasion and migration in various tumors $(18,19)$. However, the effect of Aurora-B inhibition in NSCLC malignancies remains to be fully elucidated. In the present study, to investigate the effect of Aurora-B inhibition on NSCLC cell migration and invasion, the recombinant LV targeting Aurora-B was constructed to inhibit Aurora-B expression in A549 cells. Furthermore, the migration and invasion of A549 cells was investigated by wound healing and Transwell assays, and the results revealed that the migration and invasion rate of cells was significantly lower in cells that were transfected with Aurora-B/LV than those that were transfected with $\mathrm{Neg} / \mathrm{LV}$. This indicated that the inhibition of Aurora-B may suppress A549 cell migration and invasion in vitro.

In addition, the potential molecular mechanisms associated with the inhibition of Aurora-B expression, and A459 cell migration and invasion suppression were analyzed. The role of the PI3K/Akt/NF- $\mathrm{B}$ signaling pathway in tumor cell invasion and migration was investigated (20-25). Long et al (26) demonstrated that ZM447439, an inhibitor of Aurora-B, was significantly associated with a decrease in Akt phosphorylation (at Ser473) and a decrease in the phosphorylation of its substrates, glycogen synthase kinase $3-\alpha$ and $-\beta$ (at Ser21 and Ser9) in Hep2 cancer cells. Akt is essential for NF- $\mathrm{NB}$ activation via the stimulation of the $\mathrm{I} \kappa \mathrm{B}$ kinase complex, which phosphorylates and inactivates $\mathrm{I} \kappa \mathrm{B}$, an inhibitor of $\mathrm{NF}-\kappa \mathrm{B}$. Previous studies have demonstrated that $N F-\kappa B$ upregulates MMP-9 (27) and the inhibition of NF- $\mathrm{BB}$ was found to downregulate MMP-2 (28). During the development of metastases, cancer cells must degrade the components of the extracellular matrix. MMPs, in particular MMP-2 and -9 , are markedly associated with this process due to their capacity to degrade the extracellular matrix, which promotes tumor invasion.

In the present study, PI3K, Akt, p-Akt and NF- $\mathrm{B}$ (p65) protein expression levels were detected by western blot analysis to investigate whether inhibiting Aurora-B led to a decrease in the activity of the PI3K/Akt/NF- $\kappa \mathrm{B}$ signaling pathway. The results revealed that the expression levels of the PI $3 \mathrm{~K}, \mathrm{p}-\mathrm{Akt}$ and $\mathrm{NF}-\kappa \mathrm{B}(\mathrm{p} 65)$ proteins were significantly decreased in cells that were transfected with Aurora-B/LV when compared with those in cells that were transfected with $\mathrm{Neg} / \mathrm{LV}$. These results indicated that the inhibition of Aurora-B downregulates the $\mathrm{PI} 3 \mathrm{~K} / \mathrm{Akt} / \mathrm{NF}-\kappa \mathrm{B}$ signaling pathway in NSCLC cells. In addition, western blot analysis was performed to investigate the expression levels of MMP-2 and -9 proteins. It was found that the protein expression levels were reduced as a result of Aurora-B inhibition when compared with the negative control cells, indicating that the inhibition of Aurora-B attenuates the activation of MMP-2 and -9 proteins.

In conclusion, the present study demonstrates that the inhibition of Aurora-B may suppress NSCLC cell invasion and migration via modulation of the PI $3 \mathrm{~K} / \mathrm{Akt} / \mathrm{NF}-\kappa \mathrm{B}$ signaling pathway in vitro. This indicates that targeting Aurora-B and the $\mathrm{PI} 3 \mathrm{~K} / \mathrm{Akt} / \mathrm{NF}-\kappa \mathrm{B}$ signaling pathway may present a potential treatment strategy for NSCLC.

\section{Acknowledgements}

The present study was supported by grants from Jiangxi Province Education Department of Science and Technology (grant no. GJJ12097).

\section{References}

1. Shi L, Tang J, Tong L and Liu Z: Risk of interstitial lung disease with gefitinib and erlotinib in advanced non-small cell lung cancer: a systematic review and meta-analysis of clinical trials. Lung Cancer 83: 231-239, 2014.

2. Carmena M, Ruchaud S and Earnshaw WC: Making the Auroras glow: regulation of Aurora A and B kinase function by interacting proteins. Curr Opin Cell Biol 21: 796-805, 2009.

3. Ohi R, Sapra T, Howard J and Mitchison TJ: Differentiation of cytoplasmic and meiotic spindle assembly MCAK functions by Aurora B-dependent phosphorylation. Mol Biol Cell 15: 2895-2906, 2004.

4. Wang F, Ulyanova NP, Daum JR, Patnaik D, Kateneva AV, Gorbsky GJ and Higgins JM: Haspin inhibitors reveal centromeric functions of Aurora B in chromosome segregation. J Cell Biol 199: 251-268, 2012.

5. Piekorz RP: Dissecting the role of INCENP-Aurora B in spindle assembly checkpoint function, chromosomal alignment and cytokinesis. Cell Cycle 9: 1678-1679, 2010.

6. Becker M, Stolz A, Ertych N and Bastians H: Centromere localization of INCENP-Aurora B is sufficient to support spindle checkpoint function. Cell Cycle 9: 1360-1372, 2010.

7. Li Y,Zhou W, Wei L, Jin J, Tang K, Li C, et al: The effect of Aurora kinases on cell proliferation, cell cycle regulation and metastasis in renal cell carcinoma. Int J Oncol 41: 2139-2149, 2012.

8. Qi G, Ogawa I, Kudo Y, Miyauchi M, Siriwardena BS, Shimamoto $\mathrm{F}$, et al: Aurora-B expression and its correlation with cell proliferation and metastasis in oral cancer. Virchows Arch 450: 297-302, 2007.

9. Takeshita M, Koga T, Takayama K, Ijichi K, Yano T, Maehara Y,et al: Aurora-B overexpression is correlated with aneuploidy and poor prognosis in non-small cell lung cancer. Lung Cancer 80: 85-90, 2013.

10. Hetland TE, Nymoen DA, Holth A, Brusegard K, Flørenes VA, Kærn J, et al: Aurora B expression in metastatic effusions from advanced-stage ovarian serous carcinoma is predictive of intrinsic chemotherapy resistance. Hum Pathol 44: 777-785, 2013.

11. Bonet C, Giuliano S, Ohanna M, Bille K, Allegra M, Lacour JP, et al: Aurora B is regulated by the mitogen-activated protein kinase/extracellular signal-regulated kinase (MAPK/ERK) signaling pathway and is a valuable potential target in melanoma cells. J Biol Chem 287: 29887-29998, 2012.

12. Tuncel H, Shimamoto F, Kaneko Guangying Qi H, Aoki E, Jikihara $\mathrm{H}$, Nakai $\mathrm{S}$, et al: Nuclear Aurora B and cytoplasmic Survivin expression is involved in lymph node metastasis of colorectal cancer. Oncol Lett 3: 1109-1114, 2012. 
13. Honma K, Nakanishi R, Nakanoko T, Ando K, Saeki H, Oki E, et al: Contribution of Aurora-A and -B expression to DNA aneuploidy in gastric cancers. Surg Today 44: 454-461, 2014.

14. Wang WR, Yang SS, Lin JX, Zeng ZY, Liu DM and Liu HT: Expression of Aurora-B in non-small cell lung cancer and its clinical significance. Nan Fang Yi Ke Da Xue Xue Bao 29: 1853-1856, 2009 (In Chinese).

15. Ma YX and Li XZ: Effect of aurora kinase B inhibitor AZD1152 in the treatment of cisplatin-resistant ovarian carcinoma. Zhonghua Fu Chan Ke Za Zhi 48: 46-50, 2013 (In Chinese).

16. Sak A, Stuschke M, Groneberg M, Kübler D, Pöttgen C and Eberhardt WE: Inhibiting the aurora B kinase potently suppresses repopulation during fractionated irradiation of human lung cancer cell lines. Int J Radiat Oncol Biol Phys 84 492-499, 2012.

17. Qi W, Liu X, Cooke LS, Persky DO, Miller TP, Squires M and Mahadevan D: AT9283, a novel aurora kinase inhibitor, suppresses tumor growth in aggressive B-cell lymphomas. Int J Cancer 130: 2997-3005, 2012.

18. Zhang L and Zhang S: ZM447439, the Aurora kinase B inhibitor, suppresses the growth of cervical cancer $\mathrm{SiHa}$ cells and enhances the chemosensitivity to cisplatin. J Obstet Gynaecol Res 37: 591-600, 2011

19. Pohl A, Azuma M, Zhang W, Yang D, Ning Y, Winder T, et al: Pharmacogenetic profiling of Aurora kinase B is associated with overall survival in metastatic colorectal cancer. Pharmacogenomics J 11: 93-99, 2011.

20. Lin ML, Lu YC, Chen HY, Lee CC, Chung JG and Chen SS: Suppressing the formation of lipid raft-associated Rac1/PI3K/Akt signaling complexes by curcumin inhibits SDF-1 $\alpha$-induced invasion of human esophageal carcinoma cells. Mol Carcinog 53: 360-379, 2014

21. Xu CL, Lu XL, Yan XN, Wang HL and Chen SQ: Effects of $\mathrm{PI} 3 \mathrm{~K} / \mathrm{Akt} / \mathrm{NF}-\kappa \mathrm{B}$ signal pathway on FSH facilitation on cell proliferation and invasion by human epithelial ovarian cancer. Zhonghua Fu Chan Ke Za Zhi 47: 134-138, 2012 (In Chinese).
22. Lin X, Zhang X, Wang Q, Li J, Zhang P, Zhao M and Li X: Perifosine downregulates MDR 1 gene expression and reverses multidrug-resistant phenotype by inhibiting PI3K/Akt/NF- $\kappa \mathrm{B}$ signaling pathway in a human breast cancer cell line. Neoplasma 59: 248-256, 2012.

23. Koumakpayi IH, Le Page C, Mes-Masson AM and Saad F: Hierarchical clustering of immunohistochemical analysis of the activated ErbB/PI3K/Akt/NF-kappaB signalling pathway and prognostic significance in prostate cancer. Br J Cancer 102: 1163-1173, 2010.

24. Song L, Xiong H, Li J, Liao W, Wang L, Wu J and Li M: Sphingosine kinase-1 enhances resistance to apoptosis through activation of PI3K/Akt/NF- $\kappa \mathrm{B}$ pathway in human non-small cell lung cancer. Clin Cancer Res 17: 1839-1849, 2011.

25. Cortes-Sempere M, Chattopadhyay S, Rovira A Rodriguez-Fanjul V, Belda-Iniesta C, Tapia M, et al: MKP1 repression is required for the chemosensitizing effects of NF-kappaB and PI3K inhibitors to cisplatin in non-small cell lung cancer. Cancer Lett 286: 206-216, 2009.

26. Long ZJ, Xu J, Yan M, Zhang JG, Guan Z, Xu DZ, et al: ZM 447439 inhibition of aurora kinase induces Hep2 cancer cell apoptosis in three-dimensional culture. Cell Cycle 7: 1473-1479, 2008.

27. Andela VB, Gordon AH, Zotalis G, Rosier RN, Goater JJ, Lewis GD, et al: NFkappaB: a pivotal transcription factor in prostate cancer metastasis to bone. Clin Orthop Relat Res (Suppl 415): S75-S85, 2003.

28. Felx M, Guyot MC, Isler M, Turcotte RE, Doyon J, Khatib AM, et al: Endothelin-1 (ET-1) promotes MMP-2 and MMP-9 induction involving the transcription factor NF-kappaB in human osteosarcoma. Clin Sci (Lond) 110: 645-654, 2006. 